\title{
Erfolge planen und messen
}

\section{André Peters und Steffen Brixner}

Jede soziale Einrichtung braucht ein maßgeschneidertes Controllingsystem. Mit 16 Fragen in vier Dimensionen kann dieses strategische Steuerungssystem entwickelt und etabliert werden, wie am Beispiel von stationären Pflegeeinrichtungen gezeigt werden kann.

Pflegeeinrichtungen leiden unter knappen Mitteln, starkem Wettbewerb und dynamischen Nachfrageveränderungen. Trotz sinkender Margen expandieren erfolgreiche Anbieter und erzielen attraktive Renditen. Diejenigen, die die Auslastung steigern, das Angebot verbessern und die Qualität optimieren verfügen über ein spezifisches Konzept zur Steuerung ihres Erfolges.

Die Handlungsfelder des Pflegecontrollings sind vielfältig: Einrichtungen optimieren das Leistungsangebot, die Pflegequalität und die Ausstattungsstandards. Sie verbessern den Personaleinsatz, die Mitarbeiterzufriedenheit und das Belegungsmanagement. Pflegecontrolling ist ein Steuerungssystem, mit dem die Einrichtung ihren Erfolg plant und überwacht. Sie visualisiert Informationen und bildet sie in finanziellen und potenzialorientierten Kennzahlen ab (vgl. Abb. 1).

\section{Wie man eine strategisches Steuerungssystem entwickelt}

Ausgehend von der Strategie leiten Führungskräfte je Dimension Ziele und Messgrößen der Einrichtung ab. In der Zielauswahl orientiert sie sich an den kritischen Erfolgsfaktoren der Einrichtung und lenken das Verhalten der Mitarbeitenden in die gewünschte Richtung. Über Messgrößen erhalten sie Auskunft über die notwendigen und aktuellen Zielerreichungsgrade. An monetären Kennzahlen erkennen sie, wie die eingeleiteten Maßnahmen zur Ergebnisverbesserung beitragen. Potenzialorientierte Messgrößen weisen sie auf den Aus- und Aufbau von Erfolgspotenzialen hin. Die Heimleitung stellt sicher, dass die Einrichtung auch den langfristigen Erfolg im Blick hat (vgl. Abb. 2).

In vier Dimensionen kann mit insgesamt 16 Fragen ein einrichtungsspezifisches strategisches Steuerungssystem entwickelt und erfolgreich etabliert werden.

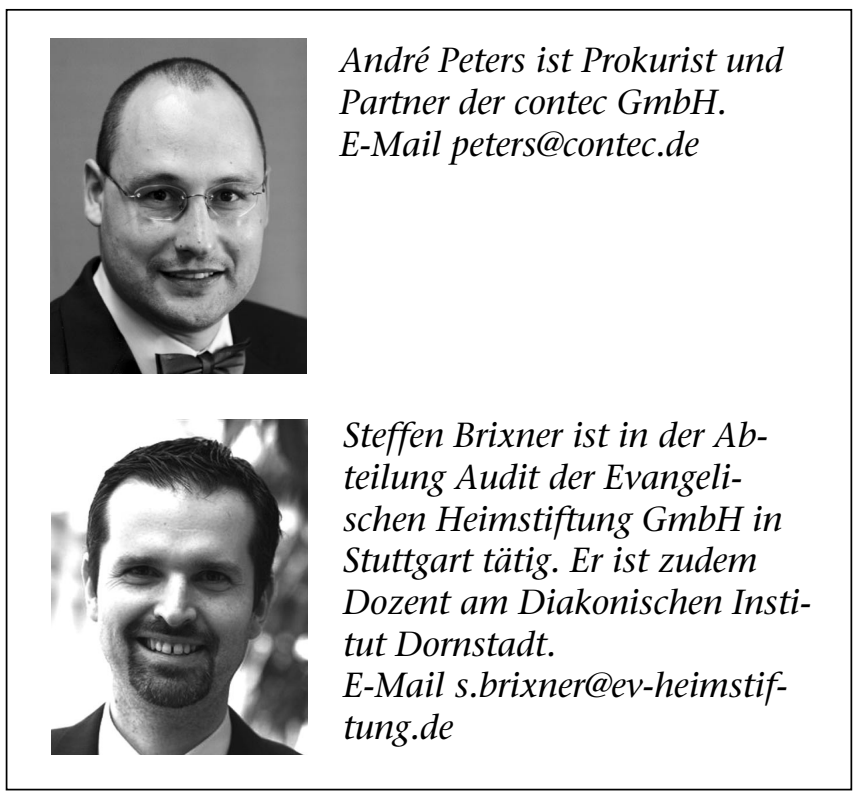

\section{Erste Dimension: Finanzen und Ressourcen}

1. Frage: Ist der lokale Markt rentabel und die Einrichtung gut im Wettbewerb positioniert?

Die Attraktivität des lokalen Marktes ergibt sich aus Angebot und Nachfrage. Während unter Investoren der Pflegemarkt global als zukunftsträchtig gilt, nehmen die tatsächlichen Überkapazitäten in vielen Regionen zu. Bei steigender Wettbewerbsintensität ist eine gute Wettbewerbsposition von Vorteil. Derjenige, der die größten Umsätze macht und über die besten Zuweiserkontakte verfügt, hat gute Chancen, lange vorn zu bleiben. Insbesondere die Kostenstrukturen sind für das Überleben entscheidend. Über eine Portfolio-Analyse bildet die Einrichtung die Marktattraktivität und ihre Wettbewerbsposition $\mathrm{ab}$.

2. Frage: Erwirtschaftet die Einrichtung die zur Rücklagenbildung notwendigen Überschüsse?

Das Betriebsergebnis dient zur Bewertung der Wirtschaftlichkeit. Im direkten Vergleich mit anderen kann die Einrichtung ermitteln, ob sie gut gewirtschaftet hat. Überschüsse sind der Garant für Investitionen. Mit einer guten Rücklagenbildung und angemessenen Eigenkapitalausstattung kann die Einrichtung auch in wirtschaftlich schwierigen Zeiten investieren. Über den Cashflow und die Eigenkapitalquote steuert die Einrichtung ihren Erfolg. 


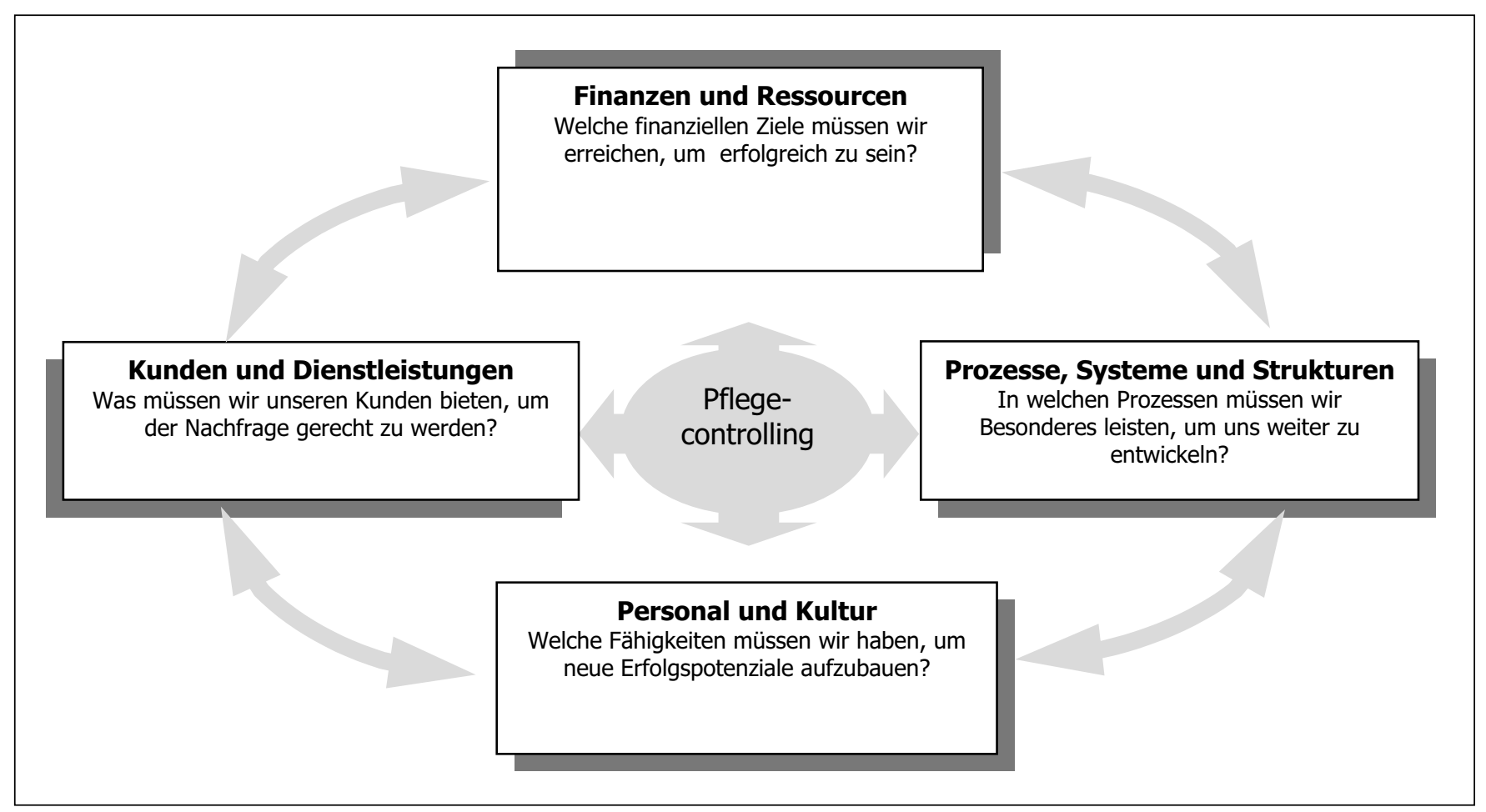

Abb. 1: Pflegecontrolling - Steuerung des Erfolges

3. Frage: Orientiert sich die Einrichtung in der Personaleinsatzplanung an Belegung und Pflegestufenverteilung?

Größter Kostenblock und wesentlicher Erfolgsfaktor in Pflegeeinrichtungen sind die Personalkosten. Sinkt die Belegung oder verändert sich die Pflegestufenverteilung, muss die Einrichtung steuernd eingreifen. Mit Modellen der kapazitätsorientierten variablen Arbeitszeit und befristeten Arbeitsverträgen passt sie die personellen Ressourcen an den Bedarf an. Sie variabilisiert ihre Fixkosten und wird weniger krisenanfällig. Die Daten zum Soll-Ist-Abgleich erhält sie aus dem Stellenplan und der Stellenbesetzung.

4. Frage: Werden Leistungen regelmäßig ausgeschrieben und indirekte Bereiche in ihrer Wertschöpfung überprüft?

Pflegeeinrichtungen haben für den Lebensmitteleinkauf und in der Ver- und Entsorgung Stammlieferanten und Hauptdienstleister. Auch in den indirekten Bereichen Reinigung, Küche, Verwaltung und Wäscherei liegen oft Einsparpotenziale. Über einen Betriebsvergleich kann das Heim seine Kosten pro Belegungstag mit den Günstigsten messen. Die höhere Gemeinkostenquote lässt Optimierungspotenziale vermuten.

\section{Dimension: Kunden und Dienstleistungen}

\section{Frage: Passen die Angebote zum Bedarf und} entwickelt die Einrichtung sie weiter?

Pflegeeinrichtungen benötigen ein Alleinstellungsmerkmal, das sich an der Nachfrage im regionalen Markt ori- entiert. Alleinstellungsmerkmale liegen in der besonderen Art eines Leistungsangebotes oder eines spezifischen Konzeptes: Einrichtungen unterscheiden sich über ihre fachliche Spezialisierung, die Biografiearbeit, das ehrenamtliche Engagement oder ein Hospizangebot. Mit pflegefachlichen Spezialangeboten wenden sie sich an junge Pflegebedürftige oder Menschen mit Behinderung. Mit einem geeigneten Raumprogramm und der fachlichen Spezialisierung der Pflegekräfte grenzt sich Einrichtung von anderen ab. Sie bewertet die Attraktivität ihres Angebotes mit einem Punktwertverfahren.

\section{Frage: Sind die Kunden und Angehörigen zufrieden?}

Ein Hauptargument für die Wahl einer Pflegeeinrichtung ist die Zufriedenheit der Bewohner. Zufriedene Pflegekunden empfehlen Einrichtungen weiter und nehmen Zusatzangebote in Anspruch. Sie sind wesentliche Werbeträger: Sie kommunizieren, »ob die Schwester Zeit hat «, »mal jemand vorliest « oder »der Geburtstag gemeinsam gefeiert« wurde. Pflegeeinrichtungen messen ihre Kundenzufriedenheit durch verschiedene Verfahren: am Ende des Aufnahmeprozesses, im täglichen Klienten- und Angehörigenkontakt, durch eine flächendeckende Befragung. Die Ergebnisse fließen im Kundenzufriedenheitsindex zusammen.

\section{Frage: Wie gut ist die Qualität der erbrachten Dienstleistung?}

Ausschlaggebendes Kriterium für die Zufriedenheit der Kunden ist die Qualität der erbrachten Dienstleistung. Die Bewertung reicht von der Strukturqualität in Einzeloder Doppelzimmern, in Gebäude und Umgebung, über die Pflege- und Betreuungsqualität bis hin zur Qualität des Essens und der Reinhaltung der Räume. Auch gemein- 
wesenorientierte kulturelle und spirituelle Angebote werden mitbewertet. Die Einrichtung entwickelt ihre Qualität über Konzepte und Standards und überwacht sie in Managementreviews, Audits und Visitationen. Heimaufsicht und Medizinischem Dienst der Krankenkassen prüfen die Einrichtung regelmäßig. Der Medizinische Dienst veröffentlicht die Ergebnisse zukünftig als Schulnoten im Internet. Die Einrichtung arbeitet Qualitätsmängeln im Beschwerdemanagement ab und dokumentiert ihre Qualitätsverbesserungen.

\section{Frage: Verfügt die Einrichtung über ein gutes Image?}

Das Image bei Ärzten, Krankenhaussozialdiensten, Beratungsstellen und Kostenträgern ist wesentlich für den Belegungserfolg der Einrichtung. Auf Festen und Veranstaltungen präsentieren sich Vertreter der Pflegeorganisation in Presse und Öffentlichkeit und bei Vereinen und Verbänden. Die Pflegeeinrichtung setzt ihre Imagemessung auf einer bestehenden Vertrauensbeziehung auf. »Critical Friends « sind bereit, auch ein negatives Feedback zu geben. Sie greift auf Experten wie Seniorenbeiräte oder Mitarbeitende in Pflegestützpunkten und Pflegeberatungsstellen zurück.

\section{Dimension: Strukturen und Prozesse}

\section{Frage: Wird die Pflege strukturiert geplant und dokumentiert?}

Um bei einer Veränderung des Pflege- und Betreuungsaufwands der Bewohner die Versorgung entsprechend schnell anzupassen, sind die Pflegeplanungen als ein Bestandteil des Pflegeprozesses zu aktualisieren. Die Anzahl der überarbeiteten Pflegeplanungen gibt Hinweise auf die Veränderungsdynamik der Bewohner. Diese quantitative Kennzahl darf jedoch nicht solitär betrachtet werden, sondern muss im Zusammenhang mit anderen Messgrößen (z. B. Einstufungs- und Höherstufungsanträge) bewertet werden.
10. Frage: Wird die Pflegequalität über Pflege- und Dokumentationsvisiten kontrolliert?

Die Pflegevisite ist ein wichtiges Instrument der Qualitätssicherung. Wesentlicher Inhalt der Pflegevisite ist neben der Prüfung der Pflegedokumentation ein Gespräch mit dem Bewohner über den Pflegeprozess und die Zufriedenheit mit den verschiedenen Leistungsbereichen. Die Teilnahme von Angehörigen an der Pflegevisite ist wichtig, um die Pflege- und Betreuungsleistungen transparent zu machen. Mit Hilfe der Pflegevisite kann die Angemessenheit, Wirtschaftlichkeit und Wirksamkeit der Pflege überprüft werden. Ziel ist es, dass für jeden Bewohner einmal jährlich eine Pflegevisite durchgeführt wird. Wichtig ist, dass die Ergebnisse der Pflegevisite (z. B. Schulungsbedarf bei der Wunddokumentation) ausgewertet und bei der Fort- und Weiterbildungsplanung berücksichtigt werden. Neben der Pflegevisite bietet sich auch die stichprobenartige Überprüfung der Pflegedokumentationen unter haftungsrechtlichen Aspekten an. Mängel in der Pflegedokumentation werden erkannt und behoben. Als Kennzahl fungiert die Überprüfungsquote.

11. Frage: Sind die Kernprozesse in Pflege, Hauswirtschaft und sozialer Betreuung beschrieben und optimiert?

Die Kernprozesse umfassen die Pflege, Betreuung und Versorgung der Bewohner. Sie beginnen mit dem Erstkontakt und enden mit dem Auszug oder Tod eines Bewohners. Über Pflege- und Betreuungskonzepte, Expertenstandards und Prozessbeschreibungen regelt die Einrichtung in welcher Abfolge welche Schritte durchgeführt werden. Sie misst ihre Qualität an definierten Prozesspunkten und optimiert die Abläufe in Qualitätszirkeln.

12. Frage: Wird ein zielgerichtetes PflegestufenControlling betrieben?

Als Kennzahl fungiert das Verhältnis von bewilligten Pflegestufenerhöhungen und der Gesamtsumme der Anträge. Mit der Kennzahl kann die Einrichtung die Qualität der Pflegedokumentation bewerten. Je aussagekräftiger und vollständiger die Pflegedokumentation, desto höher die Deckungsgleichheit zwischen tatsächlichem Pflegeaufwand und der vorliegenden Pflegestufe.

\begin{tabular}{|c|c|c|c|c|c|c|}
\hline \multicolumn{7}{|c|}{ Pflegecontrolling } \\
\hline \multirow{5}{*}{ 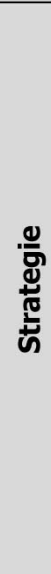 } & Dimensionen & Ziele & Messgrößen & Zielvorgabe & Maßnahmen & \multirow{5}{*}{ 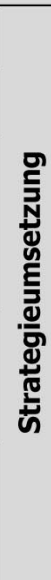 } \\
\hline & $\begin{array}{l}\text { Finanzen und } \\
\text { Ressourcen }\end{array}$ & Ertragsstärke & Betriebsergebnis & $5 \%$ Umsatzrendite & $\begin{array}{l}\text { Optimierung des } \\
\text { Kostenmanagements }\end{array}$ & \\
\hline & \begin{tabular}{|l|} 
Kunden und \\
Dienstleistungen
\end{tabular} & Kundenzufriedenheit & $\begin{array}{l}\text { Kundenzufrieden- } \\
\text { heitsgrad }\end{array}$ & $\begin{array}{l}90 \% \text { der Bewohner } \\
\text { sind zufrieden }\end{array}$ & $\begin{array}{l}\text { Erstellung eines } \\
\text { Fragebogens und } \\
\text { Befragung der Bewohner } \\
\text { drei Monate nach } \\
\text { Aufnahme }\end{array}$ & \\
\hline & $\begin{array}{l}\text { Prozesse, Systeme und } \\
\text { Strukturen }\end{array}$ & $\begin{array}{l}\text { Prozessabläufe } \\
\text { effizient gestalten }\end{array}$ & Regelungsgrad & \begin{tabular}{|l|}
$100 \%$ der \\
Schlüsselprozesse \\
sind beschrieben
\end{tabular} & $\begin{array}{l}\text { Erarbeiten von } \\
\text { Kernprozessbeschre- } \\
\text { ibungen und Einführung } \\
\text { von Expertenstandards }\end{array}$ & \\
\hline & \begin{tabular}{|l} 
Personal und Kultur \\
\end{tabular} & \begin{tabular}{|l} 
Motivierte \\
Mitarbeitende
\end{tabular} & Gesundheitsquote & $96 \%$ & $\begin{array}{l}\text { Verhältnis von } \\
\text { Anwesenheits- und } \\
\text { Arbeitstage der } \\
\text { Mitarbeitenden }\end{array}$ & \\
\hline
\end{tabular}

Abb. 2: Pflegecontrolling - von der Strategie zur Umsetzung 


\section{Dimension: Personal, Innovation und Kultur}

\section{Frage: Sind die Mitarbeitenden qualifiziert?}

Die Qualifikation der Mitarbeitenden ist ein Erfolgsfaktor der Pflegeeinrichtung. Mit ausgebildeten Wund-, Pflegestufen- und Inkontinenzexperten trägt sie zur Qualitätssicherung bei. Die Experten unterstützen die Verantwortlichen bei der Standardumsetzung. Als Inhouse-Dozenten geben sie ihr Fachwissen an die Wohnbereiche weiter. Die Einrichtung senkt ihre Kosten für externe Qualifizierungsmaßnahmen und schafft Synergien.

14. Frage: Wird eine aktive Personalentwicklung betrieben?

Mit Personalentwicklung fördern Pflegeeinrichtungen das Lernen der Organisation. Die ausgewählten Weiterbildungsthemen orientieren sich an den Einrichtungszielen. Sie berücksichtigen die Ergebnisse der Pflegevisiten, Befragungen und Audits. Eine Fortbildungsquote von 0,80 Prozent gilt als guter Zielwert. In der Quote setzt die Einrichtung die Weiterbildungstage mit den Arbeitstagen ins Verhältnis.

15. Frage: Sind die Mitarbeitenden zufrieden und motiviert?

Die Einrichtung macht die Motivation ihrer Mitarbeitenden durch die Gesundheitsquote sowie die Ergebnisse von Mitarbeiterbefragungen transparent. Eine gute Gesundheitsquote liegt bei 96 Prozent. In ihr werden die Anwesenheitstage mit den Arbeitstagen der Mitarbeitenden ins Verhältnis gesetzt. In Mitarbeiterbefragungen erhebt die Organisation alle zwei Jahre Parameter wie den Umgang mit Vorgesetzten, den Informationsfluss und die Kollegialität. Sie berücksichtigt die Rücklaufquote und veränderte Rahmenbedingungen. Drohende Kündigungen oder ausgebliebene Tariferhöhungen beeinflussen das Ergebnis.

16. Frage: Werden Wohnbereichsleitungen für Führungsaufgaben freigestellt?

Wohnbereichsleitungen sind zu einem hohen Anteil in direkten Pflegeaufgaben eingebunden. Sie können ihre Führungsaufgaben wahrnehmen, wenn die Einrichtung ihnen eine anteilige Freistellung gewährt. Als Kennzahl fungiert die Freistellungsquote. Die Einrichtung setzt den Zeitanteil für Führungsaufgaben mit der monatlichen Gesamtarbeitszeit ins Verhältnis. Mit dem Soll-Ist-Abgleich kann sie ermitteln, ob die tatsächliche Freistellungsquote mit der vereinbarten übereinstimmt.

\section{Fazit}

Die Pflegeeinrichtung bezieht die Mitarbeitenden über das Pflegecontrolling in die Weiterentwicklung der Organisation ein. Das Mitwissen der Betroffenen schafft Vertrauen und ihr Mitdenken ermöglicht Verbesserungen.

Die Führungskräfte machen ihre Ziele transparent. In kooperativen Planungs- und Auswertungsrunden stärken sie das Engagement der Mitarbeitenden und schaffen eine gute Basis für den gemeinsamen Erfolg. Sie ermöglichen gute wirtschaftliche Ergebnisse und nachgefragte Dienstleistungen.

Mit den ausgewählten Kennzahlen verfügt die Leitung über die strategisch relevanten Steuerungsinformationen. Risiken werden für sie kalkulierbarer und die Einrichtung ist auch in turbulenten Zeiten am Markt erfolgreich.

\section{Mit Patientenverfügung selbstbestimmt und in Würde sterben}

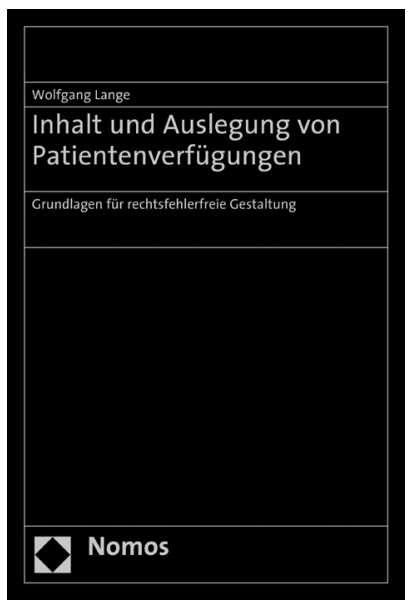

\section{Inhalt und Auslegung von Patientenverfügungen}

Grundlagen für rechtsfehlerfreie Gestaltung Von RAuN Dr. Wolfgang Lange, FAErbR

2009, 424 S., brosch., 89,- $€$,

ISBN 978-3-8329-3788-1

Das Selbstbestimmungsrecht des nicht mehr einwilligungsfähigen Patienten soll durch Patientenverfügungen gewahrt werden.

Neben der Darstellung der rechtlichen Grundlagen für Inhalt und Auslegung von Patientenverfügungen untersucht und bewertet der Autor alle verfügbaren Formulare. Darüber hinaus enthält das Werk einen Formulierungsvorschlag für eine Patientenverfügung.

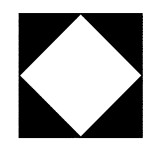

\section{Nomos}

